\title{
Quantum transport in a model of granular conductors
}

\author{
Tai-Kai Ng and Ho-Yin Cheung \\ Department of Physics, Hong Kong University of Science and Technology, Clear Water Bay Road, Kowloon, Hong Kong
}

(Received 25 April 2004; revised manuscript received 12 July 2004; published 17 November 2004)

\begin{abstract}
The problem of quantum coherent transports in granular systems is discussed in this paper with a model of disordered granular conductors, where transport properties can be computed exactly. We argue that the perturbation theory result obtained by Beloborodov et al. [Phys. Rev. B 63, 115109 (2001)] is valid for a more general class of granular systems beyond usual granular metals.
\end{abstract}

DOI: 10.1103/PhysRevB.70.172104

PACS number(s): 72.15.-v, 73.23.-b

Interest in studying electronic transports properties of granular metals has persisted for a long time..$^{1-7}$ The main difference between granular metals and normal disordered metals is that there exists a large temperature range where transports is dominated by incoherent processes usually called quantum dissipation, and the transports in this temperature range can be described by an effective action proposed first by Ambegaokar, Eckern, and Schön (AES). ${ }^{2}$ The competition between Coulomb interaction and quantum dissipation determines the electronic properties in this temperature range. However, it is also expected that coherent transports should recover in the low-temperature regime $T \leqslant \Delta \epsilon$, where $\Delta \epsilon$ is the average energy level spacing in the grains. ${ }^{3}$ A theory describing the crossover between the two regimes in granular metals have been proposed by Beloborodov et al., ${ }^{1}$ where they formulated a perturbation theory valid in the intermediate- to low-temperature regime $E_{T} \gg k_{B} T$, where $E_{T} \sim D / L^{2}$ is the average Thouless energy of grains and $L^{3}=\Omega$ is the average grain volume.

Beloborodov et al. considered a model Hamiltonian,

$$
H=\sum_{i, n}\left(\epsilon_{n}^{(i)}-\mu\right) c_{i n}^{\dagger} c_{i n}+\frac{1}{\Omega} \sum_{\langle i, j\rangle, n, m} t_{i j}^{n m}\left(c_{i n}^{\dagger} c_{j m}+\text { H.c. }\right),
$$

where the first term represents the Hamiltonian for single grains in the single-particle eigenstate representation, $\epsilon_{n}^{(i)}$,s are the eigenenergies in grain $i$, and the second term is the tunneling Hamiltonian between different grains. Notice that spin is neglected in the above Hamiltonian since it is irrelevant in the absence of magnetic field, spin-orbit coupling, and electron-electron interaction. The energy levels $\epsilon_{n}^{(i)}$ 's in different grains are random but characterized by the same energy level distribution $\rho(\epsilon)$, corresponding to finite mesoscopic systems in the weak-disorder regime when localization effect within grains are unimportant. ${ }^{5,8}$ The tunneling matrix elements $t_{i j}$ between grains are assumed to be completely uncorrelated between different grains, i.e.,

$$
\left\langle t_{i j}^{n m} t_{k l}^{p q}\right\rangle_{t} \sim|t|^{2}\left(\delta_{i k} \delta_{j l} \delta_{n p} \delta_{m q}+\delta_{i l} \delta_{j k} \delta_{n q} \delta_{m p}\right),
$$

where \langle\rangle$_{t}$ denotes disorder average over hopping. The density-density response function was computed in terms of a perturbation theory of the parameter $1 / g_{T}$ where $g_{T}=\pi^{2}|t|^{2} \nu$ is the average tunneling conductance between grains and $\nu \sim 1 /(\Delta \epsilon \Omega)$ is the grain average density of states. In the large $g_{T}$ limit they showed that the response function has the form ${ }^{1,4}$

$$
\chi(\vec{q}, \omega) \sim \frac{\nu D q^{2}}{-i \omega+\delta D q^{2}},
$$

where $D \sim g_{T} l^{2}$ is the diffusion constant for a corresponding normal dirty metal, $l \sim$ distance between grains and $\delta=\nu \Delta \epsilon$. The response function describes "quantum dissipation" in the intermediate temperature regime $T \gg g_{T} \delta$ but describes normal diffusive behavior in the low temperature limit $T<g_{T} \delta$. It suggested that the crossover from "quantum dissipation" to "quantum coherence" regime occurs at $T \sim g_{T} \delta$.

The theory of Beloborodov et al. ${ }^{1}$ depends on condition (1b) of complete decoherence between different hopping processes, which is generally believed to be correct in describing tunneling processes between metallic grains at energy $\epsilon<E_{T}{ }^{8}$ In this limit, only retraceable paths representing elastic cotunneling processes ${ }^{3}$ contributes to coherent transports. The crossover energy scale $g_{T} \delta$ arises naturally as a result.

The crossover energy scale $\sim \Delta \epsilon$ can be inferred from a more general argument in the strong disorder limit, where all states are localized. In this limit hopping conduction dominates and an electron on an occupied site would typically jump to a site with energy $\epsilon_{h}$ above it, with $\epsilon_{h} \sim\left(\xi^{d} \nu\right)^{-1}$, where $d$ is dimension of the system, $\xi$ is the localization length, and $\nu$ is the density of states. The localization effect (or quantum coherent transports) shows up only when the temperature is lower than $\epsilon_{h}$, when hopping conduction is quenched. With the assumption that localizations within grains are unimportant, we have $\xi>L$. The grain density of states $\nu$ is of order $1 /\left(L^{d} \Delta \epsilon\right)$. Therefore $\epsilon_{h} \leqslant \Delta \epsilon$, suggesting that coherent transports appear only at energy scale senergy-level spacing within grains.

The above argument suggests that the crossover behavior obtained by Beloborodov et al. ${ }^{1}$ is a general property of granular systems and is independent of the incoherent hopping condition (1b). To show that this is indeed the case we study in this paper a model of disordered grains where hopping processes are much more coherent than given by relation (1b) and transports are not dominated by retraceable paths. We find that the transport properties computed in the model is qualitatively the same as result of 
Beloborodov et al., indicating that Eq. (2) indeed applies rather generally to systems described by Hamiltonian of the form (1a).

Our model is described by a Hamiltonian of the same form as (1a) with the assumptions that (1) the hopping matrix elements are independent of level indices, i.e., $t_{i j}^{n m} \sim t_{i j}$, and (2) that all grains are identical, i.e.,

$$
H_{m}=\sum_{i, n}\left(\epsilon_{n}-\mu\right) c_{i n}^{\dagger} c_{i n}+\frac{1}{\Omega} \sum_{\langle i, j\rangle, n, m} t_{i j}\left(c_{i n}^{\dagger} c_{j m}+\text { H.c. }\right),
$$

where the grain energies $\epsilon_{n}$ 's are identical for all grains although they are still assumed to be random with density of states characterized by finite mesoscopic systems in the weak-disorder regime. Notice that assumption (1) implies that phase coherence between different hopping processes from one grain to another is maintained in our model. We shall show that transports in our model can be computed exactly with the above two assumptions.

We consider the density-density response function

$$
\begin{aligned}
\chi\left(r_{i}, r_{j}, i \omega\right)= & \sum_{\alpha, \beta, m, n} \psi_{\alpha}^{*}\left(r_{i}, m\right) \psi_{\beta}\left(r_{i}, m\right) \psi_{\beta}^{*}\left(r_{j}, n\right) \\
& \times \psi_{\alpha}\left(r_{j}, n\right) \frac{f\left(\epsilon_{\beta}\right)-f\left(\epsilon_{\alpha}\right)}{i \omega+\epsilon_{\alpha}-\epsilon_{\beta}},
\end{aligned}
$$

where $\psi_{\alpha}$ and $\psi_{\beta}$ are exact one-particle eigenstates and $\epsilon_{\alpha}$ and $\epsilon_{\beta}$ are the corresponding eigenenergies in the model. $f(\epsilon)$ is the Fermi distribution function. $r_{i}$ and $r_{j}$ are the position vectors and $n$ and $m$ are grain level indices. For the special form of Hamiltonian (3), the one-particle eigenstates have a separable form, $\psi_{\alpha}(i, m)=\psi_{k}(i) \phi_{n k}(m)$, where $\alpha=(n, k), i$ is the grain index, and $m$ is the energy level index within a grain. $\psi_{k}(i)$ represents the spatial part of the wave function and satisfies the Schrödinger equation

$$
t(k) \psi_{k}(i)=\sum_{j} t_{i j} \psi_{k}(j)
$$

where $t(k)$ are the corresponding eigenvalues. The "grain" wave function $\phi_{n k}(m)$ satisfies the Schrödinger equation

$$
\epsilon_{n k} \phi_{n k}(m)=\epsilon_{m} \phi_{n k}(m)+\frac{t(k)}{\Omega} \sum_{m^{\prime}} \phi_{n k}\left(m^{\prime}\right),
$$

with eigenvalue equation

$$
1=t(k) g_{0}\left(\epsilon_{n k}\right),
$$

where $\quad g_{0}(z)=(1 / \Omega) \Sigma_{m}\left(z-\epsilon_{m}-\mu\right)^{-1}, \quad$ and $\quad \phi_{n k}(m)$ $=\left[1 / \Omega^{1 / 2}\left(\epsilon_{n k}-\epsilon_{m}\right)\right]\left(-\partial g_{0}(\epsilon) / \partial \epsilon\right)_{\epsilon=\epsilon_{n k}}^{-1 / 2}$. We notice that the effect of hopping disorder appears only in the spatial wave function $\psi_{k}(i)$ 's, which are solutions of the Schrödinger equation for a usual disordered one-band tight-binding Hamiltonian.

Putting Eq. (5) into (4), we obtain after some algebra

$$
\begin{aligned}
& \chi\left(r_{i}, r_{j}, i \omega\right)=\sum_{k, k^{\prime}, n, n^{\prime}, \sigma}\left(\frac{t(k)-t\left(k^{\prime}\right)}{\epsilon_{n k}-\epsilon_{n^{\prime} k^{\prime}}}\right)^{2}\left(-t(k)^{2}{\frac{\partial g_{0}(z)}{\partial \epsilon}}_{\epsilon=\epsilon_{n k}}\right)^{-1} \\
& \times\left(-t\left(k^{\prime}\right)^{2} \frac{\partial g_{0}(z)}{\partial \epsilon} \epsilon=\epsilon_{n^{\prime} k^{\prime}}\right)^{-1} \\
& \times \psi_{k^{\prime}}^{*}\left(r_{i}\right) \psi_{k}\left(r_{i}\right) \psi_{k}^{*}\left(r_{j}\right) \psi_{k^{\prime}}\left(r_{j}\right) \frac{f\left(\epsilon_{n k}\right)-f\left(\epsilon_{n^{\prime} k^{\prime}}\right)}{i \omega-\epsilon_{n k}+\epsilon_{n^{\prime} k^{\prime}}} .
\end{aligned}
$$

Introducing $G(t(k), z)=g_{0}(z) /\left[1-t(k) g_{0}(z)\right]$ and $A(t(k), \epsilon)=$ $-\operatorname{Im}[G(t(k), \epsilon+i \eta)]$, we may also write

$$
\begin{aligned}
\chi\left(r_{i}, r_{j}, i \omega\right) \\
=\int \frac{d \epsilon}{\pi} \int \frac{d \epsilon^{\prime}}{\pi} \int d E \int d E^{\prime} F\left(E, E^{\prime}, r_{i}, r_{j}\right) \frac{\left(E-E^{\prime}\right)^{2}}{\left(\epsilon-\epsilon^{\prime}\right)^{2}} \\
\quad \times A(E, \epsilon) A\left(E^{\prime}, \epsilon^{\prime}\right) \frac{f(\epsilon)-f\left(\epsilon^{\prime}\right)}{i \omega-\epsilon+\epsilon^{\prime}},
\end{aligned}
$$

where

$$
\begin{aligned}
F\left(E, E^{\prime}, r_{i}, r_{j}\right)= & \sum_{k, k^{\prime}} \delta(E-t(k)) \delta\left(E^{\prime}-t\left(k^{\prime}\right)\right) \\
& \times \psi_{k^{\prime}}^{*}\left(r_{i}\right) \psi_{k}\left(r_{i}\right) \psi_{k}^{*}\left(r_{j}\right) \psi_{k^{\prime}}\left(r_{j}\right) .
\end{aligned}
$$

Notice that hopping disorder manifest itself only in the spatial part of the wave function through $F\left(E, E^{\prime}, r_{i}, r_{j}\right)$ in our model and the disorder average over hopping enters only in computing $\langle F\rangle_{t}$. $\left\langle F\left(E, E^{\prime}, r_{i}, r_{j}\right)\right\rangle_{t}=\bar{F}\left(E-E^{\prime}, r_{i}-r_{j}\right)$ is related to the imaginary part of the average density-density response function $\operatorname{Im} \chi_{0}(\vec{q}, \omega+i \eta)$ of a corresponding oneband dirty metal with eigenstates given by the Schrödinger equation (5a), ${ }^{10}$ where

$$
\operatorname{Im} \chi_{0}(\vec{q}, \omega+i \eta)=\omega \bar{F}(\omega, \vec{q}),
$$

where $\bar{F}(\omega, \vec{q})$ is the Fourier transform of $\bar{F}(\omega, \vec{r})$. Therefore, the disorder average over hopping can be performed exactly in our model if the average density-density response function in the corresponding one-band model is known. For weak disorder we have the usual diffusive form ${ }^{10}$

$$
\bar{F}(\omega, \vec{q})=\left(\frac{\partial n}{\partial \mu}\right)_{t} \frac{D q^{2}}{\omega^{2}+\left(D q^{2}\right)^{2}},
$$

valid at small $\vec{q}$ and $\omega$, where $D$ is the diffusion constant for the corresponding one-band dirty metal, and $(\partial n / \partial \mu)_{t}$ is the corresponding density of states on the Fermi surface. The strong disorder limit will be discussed later.

We next consider average over grain disorder. Before considering the disorder average we first examine the eigenvalue spectrum $\epsilon_{n k}$ and spectral function $A(t(k), \epsilon)$ of the system for a fixed grain disorder. The energy dispersion $\epsilon_{n k}$ is determined by the eigenvalue equation $(5 \mathrm{c})$. For given grain level index $n$ it describes a disordered energy band [as a function of $t(k)$ ] sandwiched between energy levels $\epsilon_{n}$ and $\epsilon_{n+1}$ (except for the lowest and highest energy level) with bandwidth of order $\Delta \epsilon$. The corresponding spectral function $A(t(k), \epsilon)$ 
consists of a sum of $\delta$-functions with energy spacing $\sim \Delta \epsilon$. Therefore the imaginary part of the density-density response function describes intraband transition for energy $\omega \ll \Delta \epsilon$ and describes interband transition when $\omega \geqslant \Delta \epsilon$. This behavior persists rigorously even after disorder average over grains, as long as all grains are identical.

The two different regimes $\omega \geqslant(\ll) \Delta \epsilon$ have to be treated separately when performing the disorder average over grains. First we consider $\omega \ll \Delta \epsilon$. In this regime only intraband transition contributes to the imaginary part of $\chi$. For states in the same band, it is easy to show using Eq. (5c) that

$$
\lim _{t(k) \rightarrow t\left(k^{\prime}\right)} \frac{t(k)-t\left(k^{\prime}\right)}{\epsilon_{n k}-\epsilon_{n k^{\prime}}} \rightarrow[t(k)]^{2}\left(-\frac{\partial g_{0}(\epsilon)}{\partial \epsilon}\right)_{\epsilon=\epsilon_{n k}} .
$$

Together with Eq. (6), we obtain

$$
\begin{aligned}
\operatorname{Im}\left\langle\chi\left(r_{i}, r_{j}, \omega \ll \Delta \epsilon\right)\right\rangle \\
\sim-\pi \sum_{k, k^{\prime}}\left\langle\psi_{k^{\prime}}^{*}\left(r_{i}\right) \psi_{k}\left(r_{i}\right) \psi_{k}^{*}\left(r_{j}\right) \psi_{k^{\prime}}\left(r_{j}\right)\right. \\
\quad \times\left[f\left(\epsilon_{n k}\right)-f\left(\epsilon_{n^{\prime} k^{\prime}}\right] \delta\left(\omega-\epsilon_{n k}+\epsilon_{n^{\prime} k^{\prime}}\right)\right\rangle,
\end{aligned}
$$

which has the same form as the density-density response function of a usual one-band dirty metal, except that the bandwidth is of order $\Delta \epsilon$ but not of order $\left|t_{i j}\right| \sim t$. Therefore, we expect that in the weak-disorder limit,

$$
\operatorname{Im}\langle\chi(\vec{q}, \omega<\Delta \epsilon)\rangle \sim\left(\frac{\partial n}{\partial \mu}\right)_{t} \frac{\omega D q^{2}}{\omega^{2}+\left(\delta D q^{2}\right)^{2}},
$$

where $\delta \sim \Delta \epsilon(\partial n / \partial \mu)_{t}$. The $\delta$ factor renormalizes both the diffusion constant $D \rightarrow \delta D$ and the density of states $(\partial n / \partial \mu)_{t} \rightarrow(1 / \delta)(\partial n / \partial \mu)_{t}$ and the renormalization effects cancel each other in the numerator.

Next we consider $\omega \geqslant \Delta \epsilon$ where interband transition dominates. The average over hopping disorder in the density response function can be performed using Eqs. (7) and (8), with $F \rightarrow \bar{F}$. The average over grain disorder can be performed by replacing $A(E, \epsilon) A\left(E^{\prime}, \epsilon^{\prime}\right) \rightarrow\left\langle A(E, \epsilon) A\left(E^{\prime}, \epsilon^{\prime}\right)\right\rangle_{g}$, where \langle\rangle$_{g}$ denotes average over grain disorder with

$$
\left\langle A(E, \epsilon) A\left(E^{\prime}, \epsilon^{\prime}\right)\right\rangle_{g} \sim\langle A(E, \epsilon)\rangle_{g}\left\langle A\left(E^{\prime}, \epsilon^{\prime}\right)\right\rangle_{g} R\left(\epsilon-\epsilon^{\prime}\right),
$$

where $R(\omega)$ is the level-level correlation function for mesoscopic grains. ${ }^{4}$ For energies $\epsilon, \epsilon^{\prime}<E_{T}$, it is believed that the grain level-level correlation function exhibits universal behavior of quantum chaos. ${ }^{4}$ In particular, our system belongs to the orthogonal ensemble and $R(\omega) \sim 1$ for $\omega \gg \Delta \omega$, and $\sim|\omega| / \Delta \epsilon$ for $\omega \sim \Delta \epsilon$. This is valid as long as $\epsilon, \epsilon^{\prime}$ describe different energy levels (interband transition). The presence of the additional interaction term $t(k)$ is not expected to modify this general behavior.
To compute $\langle A(E, \epsilon)\rangle$ we consider an average grain density of states $\langle\rho(\epsilon)\rangle_{g}=\left\langle(1 / \Omega) \Sigma_{n} \delta\left(\epsilon-\epsilon_{n}\right)\right\rangle=(\nu / \pi)\left[\gamma /\left(\epsilon^{2}\right.\right.$ $\left.\left.+\gamma^{2}\right)\right]$, and $\langle A(E, \epsilon)\rangle_{g}=\gamma \nu / \pi\left[(\epsilon-\nu E)^{2}+\gamma^{2}\right]$ correspondingly. Setting $\left\langle A(E, \epsilon) A\left(E^{\prime}, \epsilon^{\prime}\right)\right\rangle_{g}$ into Eq. (7), we obtain after some algebra,

$$
\begin{aligned}
\operatorname{Im}\langle\chi(\vec{q}, \omega)\rangle & =\left(\frac{\partial n}{\partial \mu}\right)_{t} R(|\omega|) \frac{D q^{2}}{\omega}\left[\frac{\omega^{2}+2 \gamma\left(\nu D q^{2}+2 \gamma\right)}{\omega^{2}+\left(\nu D q^{2}+2 \gamma\right)^{2}}\right] \\
& \sim\left(\frac{\partial n}{\partial \mu}\right)_{t} R(|\omega|) \frac{D q^{2}}{\omega}
\end{aligned}
$$

for small $\omega, q$. Equations (10) and (11) can be interpolated by the formula

$$
\chi(\vec{q}, \omega) \sim\left(\frac{\partial n}{\partial \mu}\right)_{t} \frac{D q^{2}}{-i \omega+\delta D q^{2}},
$$

which has the same form as the density response function (2) obtained by Beloborodov et al., in agreement with our expectation. The differences between our result and the result of Beloborodov et al. come mainly from the different density of states $\nu$ and $(\partial n / \partial \mu)_{t}$ and are not surprising in view of the rather different model we consider here.

Because of the separation between in-grain and hopping disorder in our model, our result can be generalized rather easily to the regime of strong hopping disorder. Generally speaking we find that localization is reflected in a different form of $\bar{F}(\omega, r)$, and is observable in the density-density response function only at energy scale $\omega \leqslant \Delta \epsilon$, when transports is dominated by intraband transition.

When compared with the theory of Beloborodov et al., we find that the more coherent hopping in our model renormalizes the energy levels in the grains into (disordered) bands. After renormalization, a coherent transition between different grains can occur only within the same band and we recover a picture of renormalized elastic cotunneling processes.

The assumption of identical grains can be relaxed in our model through introduction of an interaction term,

$$
V=\sum_{i, n, m} V_{n m}^{(i)} c_{i n}^{\dagger} c_{i m},
$$

where $V_{n m}^{(i)}$ is a random scattering term within grain $i$ and are completely uncorrelated between different grains. This term can be treated by the usual perturbation theory technique and we find that the conclusion we drawn from the exact model is not modified qualitatively in a lowest-order self-energy and vertex correction calculation, in agreement with the expectation that the transports behavior described by Eq. (2) is indeed a general property of granular systems, independent of the incoherent hopping condition (1b).

The authors acknowledge support from HKUGC through Grant No. HKUST6142/00P. 
${ }^{1}$ I. S. Beloborodov, K. B. Efetov, A. Altland, and F. W. J. Hekking, Phys. Rev. B 63, 115109 (2001).

${ }^{2}$ V. Ambegaokar, U. Eckern, and G. Schön, Phys. Rev. Lett. 48, 1745 (1982).

${ }^{3}$ D. V. Averin and Yu. V. Nazarov, Phys. Rev. Lett. 65, 2446 (1990).

${ }^{4}$ See also, D. S. Golubev and A. D. Zaikin, Phys. Rev. B 69, 075318 (2004), for a different model that gives similar result.

${ }^{5}$ See, for example, Quantum Mesoscopic Phenomena and Mesoscopic Devices in Microelectronics, edited by I. O. Kulik and R. Ellialtioğlu, NATO Science Series C, (Kluwer Academic, 2000),
Vol. 559.

${ }^{6}$ A. Gerber, A. Milner, G. Deutsche, M. Karpovsky, and A. Gladkikh, Phys. Rev. Lett. 78, 4277 (1997).

${ }^{7}$ O. Agam, N. S. Wingreen, B. C. Altshuler, D. C. Ralph, and M. Tinkhan, Phys. Rev. Lett. 78, 1956 (1997).

${ }^{8}$ K. B. Efetov, Supersymmetry in Disorder and Chaos (Cambridge University Press, New York, 1997).

${ }^{9}$ See, for example, N. Mott, Metal-Insulator Transition (Taylor and Francis, 1990)

${ }^{10}$ E. Abrahams, P. W. Anderson, P. A. Lee, and T. V. Ramakrishnan, Phys. Rev. B 24, 6783 (1981). 